\title{
Dynamique des épisodes pluvieux intenses sur le Sud-Est du Massif Central Aspects météorologiques et applications hydrologiques
}

\author{
The dynamics of intense rainy spells over the South-East \\ of the Massif Central - Meteorological aspects \\ and hydrological applications
}

P. Tourasse * et Ch. Obled

Groupe Hydrologie, Institut de Mécanique de Grenoble

B.P. 53X - 38041 Grenoble Cedex

La Société Hydrotechnique de France a décerné en 1981 le Prix Henri MILON à Monsieur P. TOURASSE, auteur du présent rapport, pour sa thèse de Docteur-Ingénieur, préparée sous la direction de Monsieur Ch. OBLED, sur le sujet suivant :

"Analyses spatiales et temporelles de précipitations et utilisation opérationnelle dans un système de prévisions de crues (Application aux régions cévenoles)" [1].

Cette thèse a été vivement appréciée par le Jury du Prix Henri MILON en particulier pour son originalité. La Houille Blanche félicite chaleureusement M. TOURASSE.

Le rapport ci-dessous établi sur les mêmes bases et à partir des mêmes cheminements de pensée reflète fidèlement une partie de l'œuvre couronnée par le jury du Prix Henri MILON.

\section{Introduction}

Des moyens importants ont été dégagés ces dernières années pour essayer d'améliorer la prévision des crues dans certaines zones particulièrement sensibles đu bassin méditerranéen. C'est le cas notamment de la région des Cévennes dans le sud-est du Massif Central où une opération pilote de prévision et d'annonce des crues est en cours [2].

Dans le cadre de cette opération, l'équipe Hydrométéorologie de l'Institut de Mécanique de Grenoble a d'abord orienté ses recherches vers le développement de modèles de simulation pluie-débit qui soient exploitables en temps réel. Il est apparu cependant que ces modèles étaient très sensibles à l'évaluation des lames d'eau moyennes reçues par chacun des bassins concernés et qu'il était donc important de bien connaître les caractéristiques spatiales des champs de pluie dans cette région.

Un certain nombre d'études méthodologiques ayant déjà été effectuées dans ce sens à partir de données

(*) Actuellement à la Division Technique Générale, EDF, Grenoble. d'épisodes (cf. [3] et [4]) nous avons cherché à caractériser la distribution spatiale des pluies pour des pas de temps moins importants ( 1 heure ou 3 heures) et surtout plus proches du temps de réponse des différents bassins; ceci, pour mieux cerner la structure du système pluvieux à ces échelles de temps, mais aussi pour pouvoir appréhender la dynamique du phénomène et son incidence sur le développement et l'évolution des crues.

Analyses spatiales et temporelles des champs de pluie horaire sur le Sud-Est du Massif Central

La plupart des études portant sur l'analyse fine de l'organisation des systèmes pluvieux ([5] et [6]) sont fondées sur l'interprétation de scopes radar qui permettent, à un instant donné, d'avoir une image instantanée et très détaillée du champ de précipitations.

Dans notre cas, nous ne disposions pas de données radar et il nous fallait donc travailler sur des champs déduits du réseau pluviométrique existant. C'est pourquoi, nous avons rassemblé et dépouillé à l'échelle horaire un certain nombre d'épisodes d'automne (Tableau I) sur un réseau de 58 pluviographes couvrant, 


\begin{tabular}{|c|c|c|c|c|c|c|c|c|}
\hline \multicolumn{9}{|c|}{$\begin{array}{l}\text { Tableau 1. - Principales caractéristiques de l'échantillon des épisodes analysés. Les dates des épisodes ayant fait l'objet } \\
\text { d'une cartographie systématique au pas horaire sont soulignés. (*) Les bassins concernés sont indiqués en abrégé : } \\
\text { (Ht) Hérault, (Vd) Vidourie, (Gd) Gardons, (Cz) Cèze, (Ar) Ardèche, (Ey) Eyrieux, (Ls) Loire supérieure. } \\
\text { Les valeurs des précipitations sont indiquées en mm. }\end{array}$} \\
\hline$N^{\circ}$ & Date de début & Date de fin & $\begin{array}{c}\text { Durée } \\
\text { de } \\
\text { l'épisode } \\
\text { (environ) }\end{array}$ & $\begin{array}{c}\text { Lame } \\
\text { d'eau } \\
\text { movenne } \\
\text { de } \\
\text { I'épisode }\end{array}$ & $\begin{array}{c}\text { Maximum } \\
\text { mesuré } \\
\text { sur } \\
\text { l'épisode }\end{array}$ & $\begin{array}{c}\text { Nom de la station } \\
\text { et bassin }\left(^{*}\right)\end{array}$ & $\begin{array}{c}\text { Maxi en } \\
1 \text { heure } \\
(\mathrm{mm})\end{array}$ & $\begin{array}{c}\text { Nom de la station } \\
\text { et bassin }\left({ }^{*}\right)\end{array}$ \\
\hline 1 & 19 sept. 1971 & 20 sept. 1971 & $30 \mathrm{~h}$ & 23,1 & 68,4 & St Pierreville (Ey) & 28,5 & Besseges $(\mathrm{Cz})$ \\
\hline $\begin{array}{l}2 \\
3 \\
4\end{array}$ & $\begin{array}{rr}10 \text { oct. } & 1972 \\
25 \text { oct. } & 1972 \\
4 \text { sept. } & 1972\end{array}$ & $\begin{array}{cc}21 \text { oct. } & 1972 \\
28 \text { oct. } & 1972 \\
7 \text { sept. } & 1972\end{array}$ & $\begin{array}{l}80 \mathrm{~h} \\
36 \mathrm{~h} \\
66 \mathrm{~h}\end{array}$ & $\begin{array}{r}121,4 \\
100,8 \\
85,5\end{array}$ & $\begin{array}{l}241,7 \\
246,3 \\
227,5\end{array}$ & $\begin{array}{l}\text { Loubaresse }(\mathrm{Ar}) \\
\text { Villefort }(\mathrm{Cz}) \\
\text { Valleraugue }(\mathrm{Ht})\end{array}$ & $\begin{array}{l}90,0 \\
37,0 \\
57,0\end{array}$ & $\begin{array}{l}\text { La Rouvière (Vd) } \\
\text { St Hyppolyte (Vd) } \\
\text { Ceyrac (Vd) }\end{array}$ \\
\hline $\begin{array}{l}5 \\
6 \\
7\end{array}$ & $\begin{aligned} 20 \text { sept. } & 1973 \\
1 \text { oct. } & 1973 \\
2 \text { nov. } & 1973\end{aligned}$ & $\begin{array}{cc}21 \text { sept. } & 1973 \\
3 \text { oct. } & 1973 \\
6 \text { nov. } & 1973\end{array}$ & $\begin{array}{l}34 \mathrm{~h} \\
40 \mathrm{~h} \\
90 \mathrm{~h}\end{array}$ & $\begin{array}{r}34,6 \\
135,2 \\
94,2\end{array}$ & $\begin{array}{l}272,0 \\
183,0 \\
352,0\end{array}$ & $\begin{array}{l}\text { Le Cheylard (Ey) } \\
\text { Cassagnas (Gd) } \\
\text { Villefort (Cz) }\end{array}$ & $\begin{array}{l}80,0 \\
35,0 \\
36,5\end{array}$ & $\begin{array}{l}\text { Le Cheylard (EV) } \\
\text { St Martin C. (Gd) } \\
\text { Quissac (Vd) }\end{array}$ \\
\hline $\begin{array}{r}8 \\
9 \\
10\end{array}$ & $\begin{array}{l}16 \text { sept. } 1974 \\
14 \text { nov. } 1974 \\
17 \text { nov. } 1974\end{array}$ & $\begin{array}{l}20 \text { sept. } 1974 \\
16 \text { nov. } 1974 \\
18 \text { nov. } 1974\end{array}$ & $\begin{array}{l}90 \mathrm{~h} \\
63 \mathrm{~h} \\
40 \mathrm{~h}\end{array}$ & $\begin{array}{r}148,6 \\
64,8 \\
35,3\end{array}$ & $\begin{array}{r}371,8 \\
219,0 \\
82,5\end{array}$ & $\begin{array}{l}\text { Lasalle (Gd) } \\
\text { Valleraugue }(\mathrm{Ht}) \\
\text { Soleyrol }(\mathrm{Cz})\end{array}$ & $\begin{array}{l}65,0 \\
19,0 \\
40,0\end{array}$ & $\begin{array}{l}\text { St Hippolyte }(V d) \\
\text { Ste Cecite }(G d) \\
\text { Collet de Deze }(G d)\end{array}$ \\
\hline $\begin{array}{l}11 \\
12\end{array}$ & $\begin{array}{l}14 \text { sept. } 1975 \\
29 \text { sept. } 1975\end{array}$ & $\begin{array}{l}16 \text { sept. } 1975 \\
30 \text { sept. } 1975\end{array}$ & $\begin{array}{l}56 \mathrm{~h} \\
30 \mathrm{~h}\end{array}$ & $\begin{array}{l}63,4 \\
61,9\end{array}$ & $\begin{array}{l}176,0 \\
161,5\end{array}$ & $\begin{array}{l}\text { Montelimar }(R h) \\
\text { St Martin C. (Gd) }\end{array}$ & $\begin{array}{l}70,0 \\
57,0\end{array}$ & $\begin{array}{l}\text { La Rouvière (Vd) } \\
\text { St Etienne VF (Gd) }\end{array}$ \\
\hline $\begin{array}{l}13 \\
14 \\
15 \\
16 \\
17 \\
18 \\
19\end{array}$ & 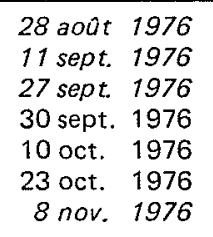 & $\begin{aligned} 31 \text { août } & 1976 \\
12 \text { sept. } & 1976 \\
29 \text { sept. } & 1976 \\
2 \text { oct. } & 1976 \\
14 \text { oct. } & 1976 \\
29 \text { oct. } & 1976 \\
11 \text { nov. } & 1976\end{aligned}$ & $\begin{array}{r}90 \mathrm{~h} \\
40 \mathrm{~h} \\
65 \mathrm{~h} \\
53 \mathrm{~h} \\
92 \mathrm{~h} \\
160 \mathrm{~h} \\
65 \mathrm{~h}\end{array}$ & $\begin{array}{r}148,0 \\
110,7 \\
57,7 \\
46,8 \\
78,8 \\
175,1 \\
180,2\end{array}$ & $\begin{array}{l}392,0 \\
400,5 \\
145,5 \\
120,7 \\
178,8 \\
404,9 \\
371,4\end{array}$ & $\begin{array}{l}\text { Villefort }(\mathrm{Cz}) \\
\text { Besseges }(\mathrm{Cz}) \\
\text { Usclades }(\mathrm{Ls}) \\
\text { Vals les Bains }(\mathrm{Ar}) \\
\text { Mt Aigoual }(\mathrm{Ht}) \\
\text { Mt Aigoual }(\mathrm{Ht} \\
\text { Villefort }(\mathrm{Cz})\end{array}$ & $\begin{array}{l}60,0 \\
85,0 \\
40,5 \\
33,0 \\
34,5 \\
23,0 \\
42,5\end{array}$ & $\begin{array}{l}\text { Malons (Cz) } \\
\text { Ste Cecile (Gd) } \\
\text { St Etienne VF (Gd) } \\
\text { Anduze (Gd) } \\
\text { St Germain (Gd) } \\
\text { Soleyrol (Cz) } \\
\text { St Etienne VF (Gd) }\end{array}$ \\
\hline $\begin{array}{l}20 \\
21 \\
22 \\
23 \\
24\end{array}$ & $\begin{array}{rr}28 \text { juil. } & 1977 \\
25 \text { aoot } & 1977 \\
5 \text { oct. } & 1977 \\
19 \text { oct. } & 1977 \\
25 \text { oct. } & 1977\end{array}$ & $\begin{array}{rr}30 \text { juil. } & 1977 \\
27 \text { aoot } & 1977 \\
7 \text { oct. } & 1977 \\
23 \text { oct. } & 1977 \\
27 \text { oct. } & 1977\end{array}$ & $\begin{array}{r}70 \mathrm{~h} \\
48 \mathrm{~h} \\
75 \mathrm{~h} \\
120 \mathrm{~h} \\
72 \mathrm{~h}\end{array}$ & $\begin{array}{r}81,8 \\
87,9 \\
121,5 \\
273,3 \\
43,7\end{array}$ & $\begin{array}{l}131,0 \\
266,5 \\
355,7 \\
596,5 \\
117,5\end{array}$ & $\begin{array}{l}\text { Villefort }(C z) \\
\text { Collet de Deze }(G d) \\
\text { Villefort }(C z) \\
\text { Ste Cecile }(G d) \\
\text { Montclus }(C z)\end{array}$ & $\begin{array}{l}19,0 \\
49,0 \\
35,2 \\
61,0 \\
30,0\end{array}$ & $\begin{array}{l}\text { Soleyrol (Cz) } \\
\text { Collet de Deze (Gd) } \\
\text { Viflefort (Cz) } \\
\text { St Etienne VF (Gd) } \\
\text { Nimes }\end{array}$ \\
\hline $\begin{array}{l}25 \\
26 \\
27 \\
28\end{array}$ & $\begin{array}{rr}4 \text { oct. } & 1979 \\
6 \text { oct. } & 1979 \\
10 \text { oct. } & 1979 \\
22 \text { oct. } & 1979\end{array}$ & $\begin{array}{rr}5 \text { oct. } & 1979 \\
7 \text { oct. } & 1979 \\
16 \text { oct. } & 1979 \\
28 \text { oct. } & 1979\end{array}$ & $\begin{array}{l}36 \mathrm{~h} \\
27 \mathrm{~h} \\
160 \mathrm{~h} \\
160 \mathrm{~h}\end{array}$ & $\begin{array}{r}50,7 \\
51,3 \\
198,6 \\
228,0\end{array}$ & $\begin{array}{l}118,0 \\
121,0 \\
566,0 \\
375,0\end{array}$ & $\begin{array}{l}\text { Uzes (Gd) } \\
\text { Soudorgues (Gd) } \\
\text { Loubaresse (Ar) } \\
\text { St Martin (Gd) }\end{array}$ & $\begin{array}{l}71,0 \\
26,0 \\
38,5 \\
16,5\end{array}$ & $\begin{array}{l}\text { La Gd Combe (Gd) } \\
\text { La Gd Combe }(\mathrm{Gd}) \\
\text { Uzes }(\mathrm{Gd}) \\
\text { Mt Aigoual }(\mathrm{Ht})\end{array}$ \\
\hline
\end{tabular}

de la région des Cévennes jusqu'à l'extrémité nord du Vivarais, une bonne partie de la bordure sud-est du Massif Central (Fig. 1). Au total, ce sont près de 2500 heures de pluie, dont certaines faibles ou quasi nulles, qui ont ainsi été traitées; soit finalement un volume considérable d'informations.

A partir de ces données, l'analyse des champs de pluie horaires a d'abord été conduite de façon purement spatiale en examinant ces champ indépendamment les uns des autres (comme autant de réalisations d'un même phénomène) et en ne retenant que les caractéristiques d'organisation du système pluvieux.

Une première analyse portant sur un sous-ensemble de 128 champs intenses a ainsi permis de montrer que la forte anisotropie, déjà mise en évidence au niveau des épisodes [4], entre la direction transversale $N W-S E$ et la direction longitudinale $S W-N E$, se conservait à l'échelle horaire; mais que la "portée" du phénomène (ou la distance d'extinction des corrélations spatiales), était beaucoup plus faible à cette échelle de temps et généralement comprise entre 10 et 30 kilomètres, soit la moitié environ des valeurs retenues pour des épisodes de 2 à 4 jours.
Néanmoins, c'est surtout la cartographie systématique d'un grand nombre de champs horaires (près de 500 cartes ont été tracées) qui nous a le mieux permis de rendre compte de ces aspects et de visualiser surtout, le degré d'organisation du système pluvieux.

A ce sujet, les conclusions les plus intéressantes concernent:

- la localisation privilégiée des fortes intensités sur une ligne $S W-N E$ parallèle à la ligne de crête principale, mais située nettement en avant de celle-ci et à une distance pouvant varier entre 10 et 30 kilomètres. En effet, s'il pleut en moyenne beaucoup moins longtemps sur l'axe Soudorgues-Malons-Montpezat que sur les zones élevées de la crête topographique, les fortes intensités y sont, par contre, beaucoup plus fréquentes; les pluies peuvent atteindre 50 ou $70 \mathrm{~mm}$ en une heure sur cet axe alors qu'elles dépassent rarement $20 \mathrm{~mm}$ en une heure à la station du Mont-Aigoual (1565 mètres) qui reçoit pourtant fréquemment, en total sur l'épisode, les quantités d'eau les plus importantes;

- l'organisation caractéristique du système pluvieux lorsqu'on observe de fortes intensités et que l'on a, 


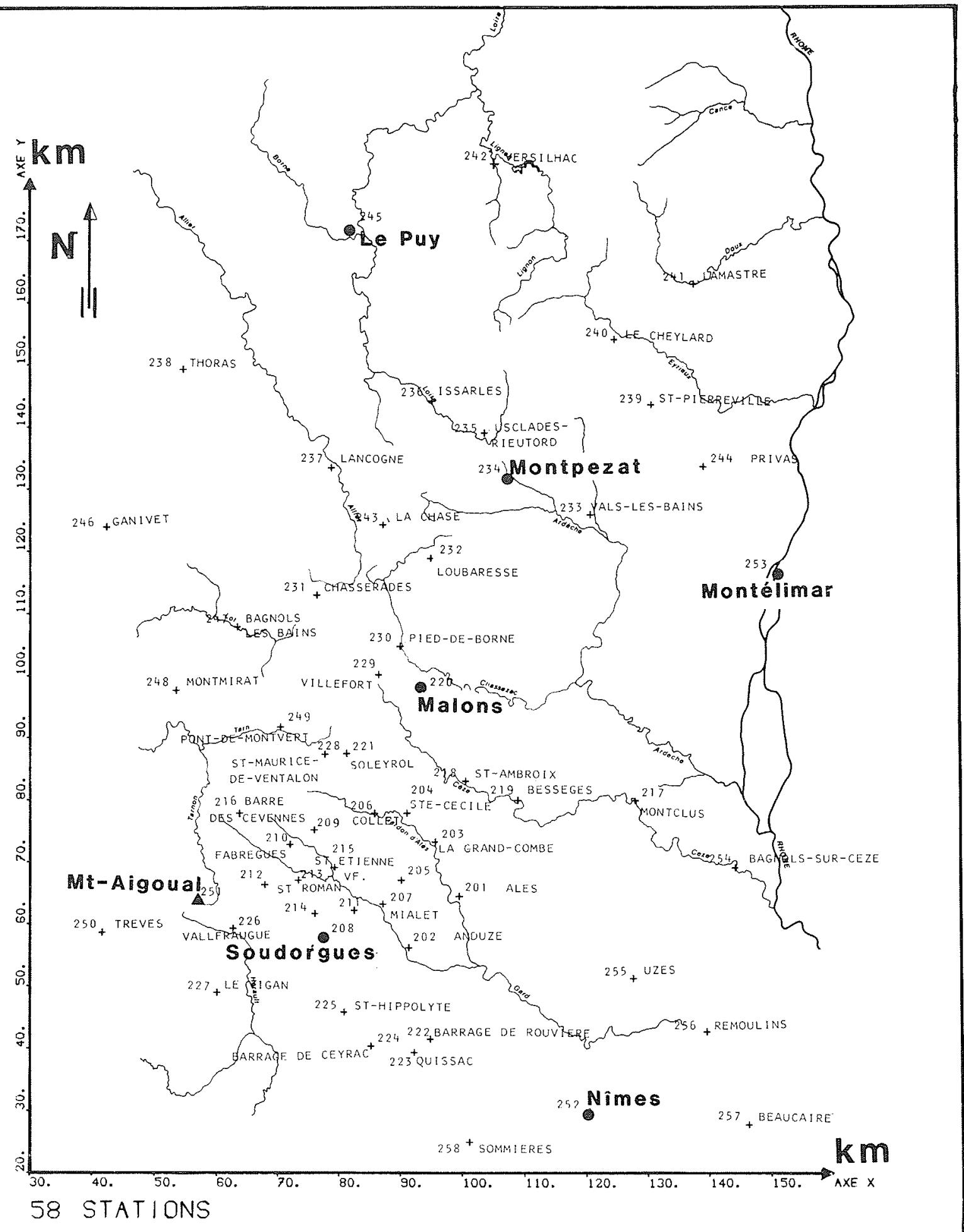

Figure 1 - Localisation des 58 stations du réseau de pluviographes.

semble-t-il, une bonne alimentation en air chaud méditerranéen. On observe alors (Fig. 2) une zone de précipitations intenses et supérieures à $10 \mathrm{~mm}$ en une heure qui est étroite et garde un peu l'allure d'une bande ;

- la présence, au sein de cette bande pluvieuse de quelques noyaux beaucoup plus intenses qui restent isolés et relativement décorrélés entre eux;
- l'existence, enfin, de gradients pluviométriques très élevés de part et d'autre de cette bande avec, dans certains cas, des pluies horaires qui passent de zéro à plus de $40 \mathrm{~mm}$ en l'espace de quelques kilomètres.

$\mathrm{Si}$ de tels résultats sont intéressants pour mieux comprendre la structure du phénomène et arriver, ainsi, à une meilleure estimation des pluies spatiales, ils sont encores insuffisants, car trop statiques, pour l'hydro- 

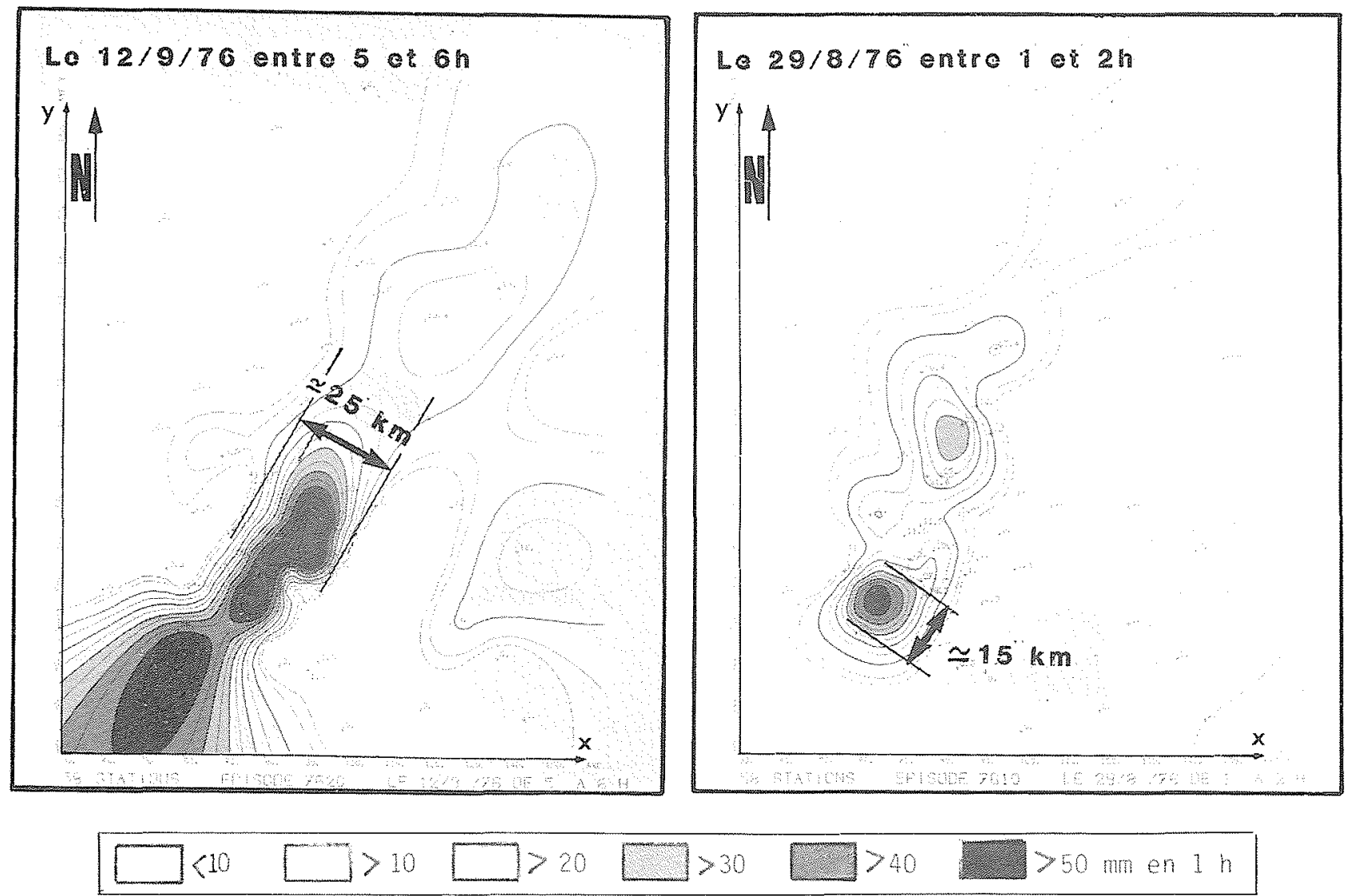

Figure 2 - Organisation du champ horaire sous la forme d'une bande pluvieuse orientée parallèlement à la ligne de crête.

logue qui s'intéresse à la prévision des crues et à leur évolution. C'est pourquoi, nous avons cherché, dans une seconde étape à introduire la composante temporelle en ne considérant plus les champs horaires individuellement mais en examinant au contraire, au sein d'un même épisode la série des cartes horaires successives.

Nous avons ainsi pu constater, sur l'ensemble des épisodes ayant fait l'objet d'une cartographie systématique (14 au total), que l'intensification des crues était due uniquement à la persistance sur place et pendant plusieurs heures de cette zone de précipitations intenses qui s'organise en travers du réseau. Il apparaît, en particulier, que le déplacement du système pluvieux parallèlement à l'axe du réseau hydrographique a peu d'incidence sur l'évolution des crues. Au contraire, lorsque nous avons pu mettre en évidence un tel déplacement, nous avons constaté qu'il se faisait soit très rapidement en fin d'épisode, après un stationnement prolongé du système pluvieux sur le relief, soit comme ce fut le cas pour l'épisode du 30 septembre 1975 (Fig. 3) pendant toute la durée de l'épisode, mais sans permettre alors la concentration de volumes importants sur les différents bassins.

Finalement, cette analyse qualitative des cartes de pluie horaires a montré :

- que les pluies commencent par apparaître sur le relief de façon un peu aléatoire en gardant, pendant cette phase transitoire qui peut être plus ou moins durable, des valeurs relativement faibles (quelques millimètres en une heure);
- puis s'organisent assez brutalement sous la forme d'une bande pluvieuse qui s'étend, en avant de la crête, sur toute l'étendue du massif et qui contient surtout un certain nombre de noyaux isolés très intenses. Au niveau de ces noyaux, les pluies peuvent dépasser $50 \mathrm{~mm}$ en une heure sur quelques dizaines, voire quelques centaines de $\mathrm{km}^{2}$, et atteindre ponctuellement des valeurs supérieures à $80 \mathrm{~mm}$ en une heure ronde Durant cette phase le système pluvieux, dans son ensemble, reste stationnaire et rend parfaitement compte d'un phénomène générateur durable et bien localisé dans l'espace; l'orographie joue alors un rôle essentiel. C'est à ce moment-là que les volumes les plus importants peuvent se concentrer et c'est bien souvent en fonction de la durée de cette seconde phase (plus de 24 heures au cours de l'épisode du 23 octobre 1977 qui fut particulièrement intense) que le caractère violent de la crue va s'affirmer ou non;

- enfin, dans un dernier temps et sous l'effet d'une modification brutale des conditions météorologiques, le système pluvieux se trouve animé d'un rapide mouvement vers l'est qui entraîne l'arrêt brusque des précipitations sur le relief.

La durée de ces différentes phases paraît assez aléatoire et de toute façon très difficile à prévoir. Elle est essentiellement liée à l'évolution, externe au système pluvieux, des conditions synoptiques et seule une analyse détaillée de la situation météorologique et de son évolution permet alors de bien comprendre cette dynamique. 

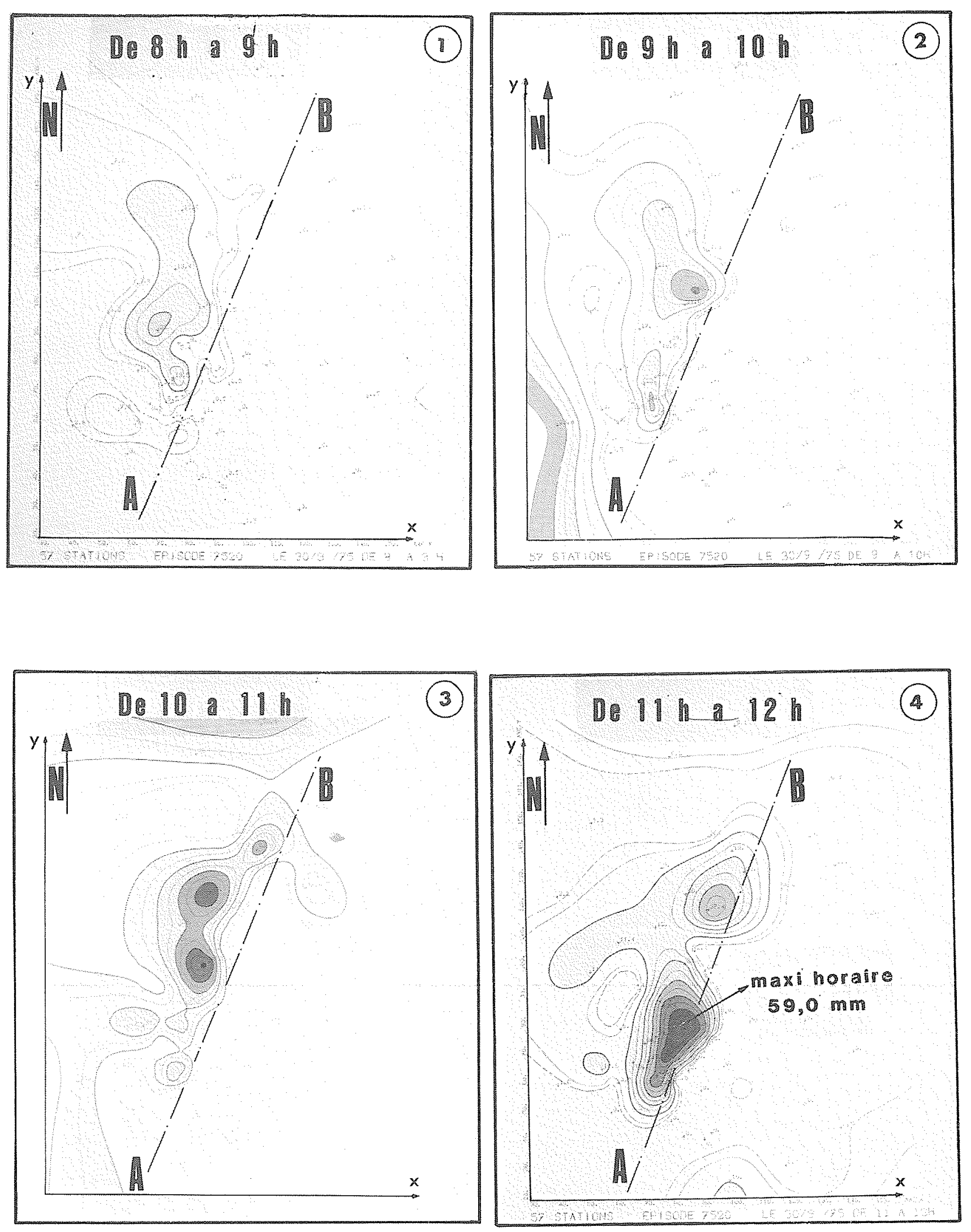

$\square<10 \square>10 \square>20 \square>30 \square>40 \square>50$ mm en $1 \mathrm{n}$





Figure 3 - Evolution du système pluvieux au cours de l'épisode du 30 septembre 1975. La ligne $A B$ figure un axe Soudorgues-MalonsMontpezat décalé par rapport à la crête et selon lequel s'orientent fréquemment les bandes de pluie qui s'organisent sur le relief. La zone pluvio-orageuse qui aborde le réseau par l'ouest (1) déborde la ligne de crête à partir de 9 heures le 30 septembre (2) et traverse rapidement l'ensemble du réseau $(4,5,6)$ pour n'affecter en $(8)$ que la vallée du Rhône. On peut noter l'importance des gradients pluviométriques à l'avant de la zone pluvieuse entre 11 et 12 heures (4). 


\section{Aspects météorologiques}

L'analyse des cartes météorologiques disponibles nous a permis, dans un premier temps, de situer le système pluvieux et ses principales structures par rapport aux éléments synoptiques. Nous avons pu montrer en particulier, et malgré l'incidence des effets orographiques qui sont souvent prépondérants dans la région, que les structures de moyenne échelle que nous avions identifiées s'orientaient parallèlement au front et à l'avant de celui-ci (Fig. 4); ce qui, dans certains cas, peut même les amener à s'orienter perpendiculairement à la ligne de crête.

Néanmoins, la prise en compte de l'ensemble de l'information météorologique existante (cartes synoptiques en altitude toutes les 12 heures et au sol toutes les 3 heures, cartes des masses d'air et comptes rendus aérologiques de Nìmes) montre que c'est surtout au niveau de l'évolution temporelle du champ que l'influence des facteurs synoptiques est déterminante; de faibles variations au niveau des configurations se traduisant, en effet, par des modifications importantes de l'organisation et de l'évolution des précipitations.

Ainsi, l'absence d'alimentation en air chaud d'origine méditerranéenne se traduit-elle par une activité beaucoup plus réduite au niveau du système pluvieux; par suite de l'orientation générale du flux en altitude au sud ou au sud-ouest, les pluies s'organisent bien sur le relief mais elles restent modérées; l'instabilité est alors moins importante et peu de noyaux intenses apparaissent alors au sein de la zone pluvieuse.

Pour d'autres configurations, au contraire, l'advection méditerranéenne est telle que l'air froid d'origine polaire maritime associé à la perturbation se trouve rejeté loin en arrière (bien au delà du Massif-Central au cours de l'épisode du 29 août 1976) permettant ainsi à des pluies d'origine purement orographique de se développer et de persister sur le relief pendant plus de 24 heures au cours de ce même épisode. L'étude détaillée de l'évolution des masses d'air met alors en évidence [7] le très net réchauffement des basses couches et des couches supérieures $\left(\Theta_{w}^{\prime} \# 20^{\circ} \mathrm{C}\right.$ au sol le 29 août 1976 à 12 heures $\Theta_{w}^{\prime}>16^{3} \mathrm{C}$ au niveau 700 mbar le 30 août 1976 à 12 heures) ainsi que la forte instabilité convective de l'air chaud d'origine méditerranéenne.

Enfin, contrairement à ce qui a parfois été avancé, des précipitations très intenses peuvent également se produire sur une grande étendue sans que l'on observe un phénomène de "blocage" au niveau des Cévennes. L'avancée de l'air froid provoque alors un soulèvement brutal de l'air chaud antérieur et donc de fortes pluies d'origine frontale (plus de $50 \mathrm{~mm}$ en une heure dans le cas de l'épisode du 30 septembre 1975); mais l'absence de blocage fait que ces pluies sont de courte durée (Cf. Fig. 3) et qu'elles ne provoquent en conséquence que des crues modérées.

Dans l'ensemble, la revue systématique des situations météorologiques concomitantes a surtout permis de montrer que les précipitations les plus intenses qui se développent sur le sud-est du Massif-Central ne résultent pas vraiment d'une situation "type" bien définie, mais plutôt d'un ensemble de facteurs favorables qui, pour une configuration donnée, influent de façon déterminante sur l'évolution du système pluvieux. Parmi ceux-ci et en mettant à part l'orientation au sud ou au sud-ouest du flux en altitude qui se trouve être une
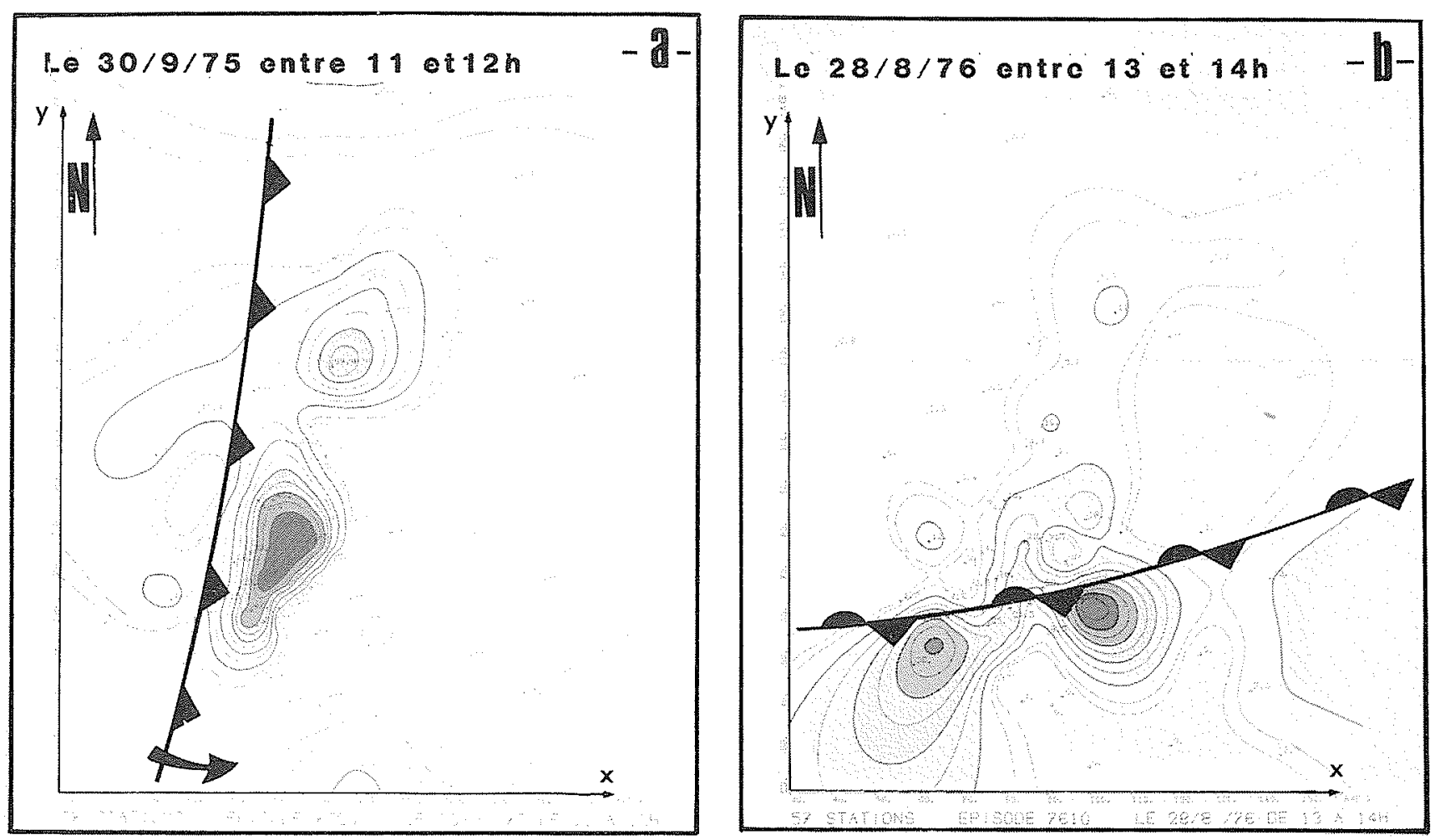

Figure 4 - Liaison avec les facteurs synoptiques. Organisation du système pluvieux au niveau d'un front. 
constante pour l'ensemble des situations que nous avons analysées, il convient de mentionner : l'intensité de ce flux; l'importance de l'advection méditerranéenne à l'avant du front froid; la nature et l'importance de l'instabilité dans l'air chaud; l'orientation générale du front froid et l'existence, ou non, à son niveau, de creusements secondaires; et surtout enfin, l'évolution de l'air froid postérieur dont le blocage au niveau des Cévennes s'accompagne d'une persistance de l'activité pluvieuse sur le relief et peut même, dans certains cas, être à l'origine d'une nouvelle cyclogénèse (par exemple, quand une goutte froide vient s'isoler aux abords de la péninsule ibérique) mais dont l'avancée peut également provoquer l'évacuation rapide du système pluvieux au-delà des Cévennes.

\section{Conclusion}

L'intérêt de cette étude repose essentiellement sur l'originalité de l'information utilisée (les cartes de pluie horaire) qui nous a permis, grâce aussi à l'interprétation conjointe des champs météorologiques, de mieux comprendre le mécanisme des précipitations dans cette région du sud-est du Massif-Central.

Outre une meilleure connaissance des caractéristiques spatiales des champs de pluie horaire, elle rend compte de certains aspects qu'il était impossible d'appréhender jusqu'alors en travaillant à un pas de temps plus important.

En privilégiant, par ailleurs, une approche plutôt climatologique par rapport à l'analyse détaillée d'un cas d'espèce, nous espérions également pouvoir tester la validité "statistique" des conclusions avancées. Malheureusement, le nombre de cas étudiés est peut-être encore trop faible pour pouvoir véritablement juger de la robustesse de celles-ci, et seule la poursuite (d'ailleurs en cours) de cette étude sur d'autres cas permettra finalement de le faire.

\section{Remerciements}

Cette étude a été menée à bien dans le cadre de l'action de la D.G.R.S.T. "Maîtrise de l'eau dans les bassins méditerranéens" et grâce à l'aide financière de la Direction Départementale de l'Equipement du Gard à Nimes.

Les données utilisées ont été mises à notre disposition par les services de la Météorologie Nationale à Grenoble et à Lyon; de la Division Technique Générale d'Electricité de France à Grenoble et de la Direction Départementale de l'Equipement du Gard à Nîmes.

\section{Références}

[1] TOURASSE P. - Analyses spatiales et temporelles de précipitations et utilisation opérationnelle dans un système de prévision de crues. Thèse de Docteur-Ingénieur, Université Scientifique et Médicale de Grenoble, mars 1981.

[2] BILLUART M., TOURASSE P. - Telemetering and simulation technique for a complete real time flood forecasting system, bulletin AISH $n^{\circ} 129$, colloque d'Oxford (GB) sur La prévision hydrologique, avril 1980 , p. 3-9.

[3]CREUTIN J.D., OBLED Ch. - Objective analyses and mapping technique for rainfall fields; an objective comparison, Water Ressources Research, à paraitre, 1982.

[4]CREUTIN J.D. -- Méthodes d'interpolation optimale de champs hydrométéorologiques. Comparaison et application à une série d'épisodes pluvieux cévenols. Thèse de DocteurIngénieur, Université Scientifique et Médicale de Grenoble, décembre 1979 .

[5] HOBBS M. al. - The mesoscale and microscale structure and organisation of clouds and precipitations in midlatitude cyclones. A case study of a cold front, J. Atm. Sciences, 1980. Vol. 37 , pp. 568-596.

[6] JAMES P.K., BROWNING K, - Mesoscale structure of line convection at surface cold fronts, Quart. J.R. Met. Soc., 1979. Vol. 105, pp. 371-382.

[7] THALAMY J. - Etude détaillée de quelques situations météorologiques ayant provoqué des crues sur les Cévennes. Rapport de stage, Ecole Nationale de la Météorologie. Institut de Mécanique de Grenoble, mai 1981. 


\section{Discussion}

Président: M. R. DAR VES-BORNOZ

\section{Le président. - Y a-t-il des questions?}

Mme DACHARR Y. - I faut se garder de parler de "situations météorologiques cévenoles". Les causes pluviales des crues cévenoles relèvent, certes, de situations à peu près identiques en surface et en altitude, aujourd'hui bien connues (P. PAUL, 1969 et M. DACHARRY, 1974) mais l'affrontement des masses d'air, générateur de fortes pluies frontales, peut se produire plus ou moins près des Cévennes (ex. sur le Haut Limousin en 1960, à I'avant des Cévennes en 1958). Quand il est situé sur les Cévennes, à l'ascendance cyclonique s'ajoute l'ascendance forcée au franchissement de l'obstacle orographique et les abats d'eau sont considérables sur toutes les stations "au vent" des versants perpendiculaires au courant perturbé.

M. MASSON. - Toutes les études spatiales sur les précipitations à un intervalle de temps fin soulignent la difficulté d'obtenir des mesures synchrones. Quelle précision estimez vous avoir dans le synchronisme des mesures utilisées?

M. TOURRASSE. - Pour l'étude de ce réseau, nous disposions d'études de services différents. Les données que nous avons utilisées provenaient de trois organismes : le Service d'équipement du Gard à Nîmes, qui gère la partie du réseau située sur la partie cévenole; d'autre part les services de lEDF (de la DTG), qui gère la pertie située sur les bassins de l'Ardèche, $d u$ Doux et de l'Eyrieux; enfin le Service météorologique, dont nous avons obtenu les données des stations de l'Aigoual, de Montélimar et du Puy. Je reconnais que cela peut-être une source supplémentaire de difficultés que de récolter des données provenant d'organismes différents.

La première chose à dire est que nous avons travaillé sur des données horaires, parce que nous estimions qu'il n'était pas envisageable de travailler à des pas de temps fin, compte tenu précisément des problèmes de décalage. Nous ne nous faisons pas d'illusions, il y a sûrement des décalages de l'ordre du quart d'heure sur certains pluviographes, et, par conséquent, travailler à des pas de temps plus fins n'aurait pas été sérieux. A l'échelle horaire, ce qui nous a finalement confortés sur la qualité des données dont nous disposions, c'est la relative cohérence spatiale des résultats que l'on obtenait.

Si l'on a $50 \mathrm{~mm}$ en une seule station et qu'à côté il n'y a rien, on peut commencer à remettre en question les résultats de cette station. En cartographiant le champ de précipitations, on avait une bonne idée de la cohérence spatiale du phénomène. Sur la carte que je vous ai montrée sur un transparent, on voyait nettement que toute la zone des précipitations importantes était confortée par un grand nombre de stations témoins à l'intérieur de cette zone et qu'à l'avant de la zone des précipitations importantes, on a un nombre important de pluviographes qui indiquaient une précipitation égale à 0 . C'est un peu cette cohérence entre les différentes stations qui nous a permis de penser qu'il y a parfois des problèmes un peu surprenants.

M. OBLED. - Ayant participé à cette étude avec $\mathrm{M}$. TOURASSE, je pense que l'on peut insister un peu plus sur certaines conclusions obtenues.

D'abord, ces études nous ont permis d'éliminer certaines hypothèses avancées dans les milieux hydrologiques. Par exemple on avait avancé, avec une certaine logique, que les crues étaient dûes au déplacement du système pluvieux parallélement à l'axe de la rivière, de l'amont vers l'aval d'où un raidissement de l'onde de crue. Or la cartographie systématique des pluies nous a montré que climatologiquement, c'est-à-dire pour un nombre important d'épisodes pluvieux, les crues étaient dûes uniquement à la persistance du système pluvieux là où il était apparu.

D'autre part, sur l'aspect météorologique, on peut dire que les pluies intenses sont liées d'abord à un effet orographique et éventuellement à l'instabilité de l'air chaud. Mais cette étude nous a permis de voir que la masse d'air froid, ou le grand froid, peut se trouver très loin de la région cévènole bien que celle-ci reçoive des pluies de l'ordre de $50 \mathrm{~mm} / \mathrm{h}$.

A ce propos, je dirais que lorsque l'on atteint la gamme des 50 à $60 \mathrm{~mm} / \mathrm{h}$, des incertitudes de $10 \mathrm{~mm} / \mathrm{h}$ n'ont guère d'importance. On peut se satisfaire d'une relative mauvaise qualité des données, à condition de préserver une cohérence spatiale raisonnable.

Pour revenir aux aspects météorologiques, il arrive cependant que le front froid s'approche et ensuite traverse la région. C'est même ainsi que l'on décrit parfois la situation cévenole type. Le passage du front provoque parfois une intensification, ou au moins un type de précipitations correspondant à la durée de ce passage. Mais les intensités restent tou tefois du même ordre que précédemment ce n'est pas un pic qui va les multiplier par deux. Et, à l'inverse, le passage du front froid a très souvent l'avantage d'annoncer la fin des précipitations intenses.

Voilà un certain nombre de remarques que je voulais faire.

Je voudrais maintenant poser à $M$. ROUSSEAU, qui est présent, une question sur le plan des méthodes d'analyse des situations météorologiques associées. La compréhension de ces phénomènes présente, outre leurs incidences économiques, un intérêt scientifique certain. Mais le manque de données sur la région est assez critique et les stations de radio-sondage de Bordeaux, Lyon, Nîmes et Milan suffisent à peine à la cerner. Il existe certes des cartes analysées tracées manuellement par les prévisionnistes. Mais pensez-vous que le modèle à mailles fines que vous avez décrit hier, activité au pas de l'heure (ou de 3 heures) permettrait une meilleure définition de la situation météorologique et de son évolution?

M. ROUSSEAU. - Je pense en effet que notre connaissance évidemment très bonne du relief permet d'améliorer la définition de la situation météorologique. Je reconnais que nous ne sommes pas encore actuellement tout à fait à l'échelle du phénomène, mais avec notre modèle à maille fine, qui est d'ailleurs utilisé tous les jours, le phénomène cévenol est perçu. Au sujet de cette situation de septembre, deux aspects sont à souligner. Du point de vue synoptique il y a eu, du fait de conditions à grande échelle, le blocage d'un front froid très actif, qui est resté dans une zone de $1000 \mathrm{~km}$ pendant 4 jours, ce qui est déjà assez rare. D'autre part les caractéristiques du relief ont amplifié localement les précipitations de façon considérable.

Je crois qu'il serait très utile de faire des simulations des phénomènes exceptionnels de ce type ; mais alors il serait encore mieux de travailler sur une maille encore plus fine que celle sur laquelle je travaille actuellement.

M. OBLED. - Je le pense aussi.

Je pense que M. ROUSSEAU a relativement bien répondu à ma question. D'après lui, l'aspect orographique peut être résolu par le modèle. Je voudrais savoir si, du point de vue météorologique, on sait comment une masse d'air froid franchit un relief et,en particulier, dévale au côté sous le vent. Ceci, parce que, dans un tiers des cas, comme l'a montré M. TOURRASSE, les perspectives que nous avons avancées sont limitées par un faible nombre de cas, une bonne vingtaine que nous espérons réussir à maintenir en routine pour un certain suivi de cette étude. Dans un tiers des cas, il y a passage visible, sensible du front froid et accroissement sensible des intensités de l'ordre de 30 à $50 \%$. Donc, il semble bien que l'on connaisse l'importance de ce phénomène que les météorologistes comprennent bien, et l'on peut, dans ce cas, bénéficier de leurs connaissances.

Le Président. - Je m'excuse d'être obligé d'arrêter ici cette discussion, mais vous pouvez la continuer ensemble. Je crois d'ailleurs que ces discussions ne se limiteront pas à ces journées et que nous aurons certainement à les reprendre ultérieurement. 


\section{Abstract \\ The dynamics of intense rainy spells over the South-East of the Massif Central - Meteorological aspects and hydrological applications}

The whole south-eastern side of the Massif Central is regularly subject, in particular in Autumn, to periods of very intense rainfall $(682 \mathrm{~mm}$ of rain in $48 \mathrm{~h}$ at the Mont-Aigoual on one occasion, on October $31 \mathrm{st} 1963 ; 85 \mathrm{~mm}$ in exactly an hour in three different stations on September 12 th, 1976 etc.), which can cause violent and sometimes catastrophic floods in some basins.

For a better understanding of the phenomenon and to obtain a closer view of the spatial characteristics of rainfall fields in the region, data on all intense rainfall periods from 1971 to 1979 were compiled and analysed, in hourly steps, using a network of 58 rainfall recording stations, i.e. a total of approximately thirty different episodes.

Systematic plotting of the most interesting episodes revealed that during phases of high activity, rainfall had the very specific shape of a narrow rainy strip (a few kilometers wide), parallel to the topographical crest, but located distinctly in front of it at a distance of approximately twenty kilometers. The strip contains very intense nuclei within which rain frequently exceeds $50 \mathrm{~mm}$ an hour. These nuclei can cover a few dozen to a few hundred square kilometers.

Joint analysis of hourly rainfall graphs and the concomitant meteorological fields has allowed for better understanding of the phenomenon, as well as the relationships which can exist between the main synoptic factors (intensity of flow, position of the front, etc.), and the organisation of the rainfall field.

It was thus ascertained that the rainfall drip developing over the mountainside did not (except for some special cases or at the end of a rainy episode, following a sudden advance of the front) move systematically in a single direction, but that the rainfall system, once organised, remained quite stable, without any general movement. Sharp variations in local intensity can nevertheless be observed, associated with the magnitude of convective activity, making the phenomenon one of rather intermittent character.

It should also be said that the study has not been completed, and is being continued with a larger volume of information : strengthening the network better to understand the situations in some zones, and taking the greater number of episodes into consideration. 